\title{
ESTUDO ETNOBOTÂNICO QUALITATIVO COM PROFISSIONAIS E USUÁRIOS DO SISTEMA ÚNICO DE SAÚDE DE JUÍNA - MT, BRASIL
}

\author{
Rodrigo Silva Rios ${ }^{1}$, Leda Maria De Souza Villaça', Suzy Helen Dourado², Silvio Carlos \\ Bieski $^{3}$ e Isanete Geraldini Costa Bieski ${ }^{4}$ \\ 15ecretaria de Saúde de Juína, Brasil, rodrigorios_cast@hotmail.com, ledavillaca@hotmail.com \\ ${ }^{2}$ Universidade Federal do Mato Grosso, Brasil, suzyhellen1@hotmail.com \\ ${ }^{3}$ Faculdade Noroeste de Mato Grosso, Brasil, silviobieski11@gmail.com \\ ${ }^{4}$ Instituto do Saber Ativo, Brasil, isabieski20@gmail.com
}

\begin{abstract}
Resumo. O ser humano sempre estabeleceu uma íntima relação com plantas medicinais para o tratamento de doenças. O objetivo foi analisar a percepção de enfermeiros e usuários de Estratégias de Saúde da Família (ESF) Juína-MT, sobre a Fitoterapia.Utilizou-se o estudo de corte transversal, com intuito de coletar informações, possibilitar a descrição do conhecimento, com amostragem aleatória simples, por sorteio. Com as perguntas: você considera a fitoterapia uma prática importante de uso? Com quem aprendeu sobre o uso da planta medicinal? Qual (is) planta (s) medicinal (is) você conhece? Como obtém a planta medicinal para uso? A pesquisa ocorreu de set a out/2017, aprovado no Comitê de Ética. As informações obtidas apontaram que a maioria dos participantes utilizam plantas medicinais, se mostrando favoraveis a inserção da fitoterapia no Sistema Unico de Saúde (SUS), a maioria aprederam sobre fitoterapia nas raizes familiares. As plantas medicinais mais utilizadas são preferencialmente Allium sativum L..(alho) e Fredericia chica (Bonpl.), crijiru, para infeções urinárias, rins, hipertensão e diabetes. O conhecimento etnobotânico muito contribui com a efetivação das políticas nacionais e estadual de plantas medicinais e fitoterápicos no SUS, assegurando a efetivação da promoção, proteção e recuperação da saúde da população de forma segura e eficaz.
\end{abstract}

Palavras-chave: Fitoterapia; Plantas Medicinais, Pesquisa Qualitativa; Prevenção

QUALITATIVE ETHNOBOTANICAL STUDY WITH PROFESSIONALS AND USERS OF THE UNIFIED HEALTH SYSTEM OF JUÍNA - MT, BRAZIL

\begin{abstract}
The human being has always established an intimate relationship with medicinal plants for the treatment of diseases. The objective was to analyze the perception of nurses and users of Family Health Strategies (FHS) Juina-MT, about Phytotherapy. The cross-sectional study was used in order to collect information, enable the description of knowledge, with sampling simple random, by lot. With the questions: do you consider phytotherapy an important practice of use? With whom did you learn about the use of the medicinal plant? Which medicinal plant (s) do you know? How do you get the medicinal plant for use? The research took place from Sep to Oct / 2017, approved by the Ethics Committee. The information obtained showed that the majority of the participants use medicinal plants, showing that the insertion of phytotherapy in the Unified Health System (SUS) was favorable, the majority learned about phytotherapy in the family roots. The most used medicinal plants are preferably Allium sativum L .. (garlic) and Fredericia chica (Bonpl.), Crijiru, for urinary infections, kidneys, hypertension and diabetes. Ethnobotanical knowledge greatly contributes to the effectiveness of national and state policies on medicinal and herbal plants in SUS, ensuring the effectiveness of the promotion, protection and recovery of the population's health in a safe and effective manner.
\end{abstract}

Keywords: Phytotherapy; Medicinal Plants, Qualitative Research; Prevention. 


\section{INTRODUÇÃO}

Desde os primórdios dos tempos, o ser humano tem estabelecido íntima relação com os reinos vegetal e animal para o tratamento de suas enfermidades (Machado, 1945; Matos, 1985). Estudos arqueológicos demostram que a referência mais antiga que se tem conhecimento do uso das plantas medicinais datam de mais de sessenta mil anos. As primeiras descobertas foram feitas por estudos arqueológicos em ruínas do Irã, também na China, em 3.000 a.C., já existiam farmacopeias que compilavam as ervas e as suas indicações terapêuticas (Rezende \& Cocco, 2002; Khan \& Ali, 2015).

O Brasil é um país de grande diversidade biológica e cultural, por isso, as pessoas apresentam considerável conhecimentos e tecnologias tradicionais, onde com o passar dos anos vem evoluindo e passando de geração em geração, entre os quais se destaca o vasto acervo de saberes sobre o manejo, o cuidado e utilização de plantas medicinais (Battisti et al., 2013).

Dados da Organização Mundial da saúde, estimam que $80 \%$ da população mundial utilizam da prática de medicina tradicional nos cuidados básicos de saúde (Brasil, 2006a). A fitoterapia sobreviveu no Brasil devido às raízes profundas na consciência popular que reconheceu sua eficácia e legitimidade. Essa terapia é regulamentada pela Resolução de Diretoria Colegiada (RDC) $n^{0}$ 26/2014 que dispõe sobre o registro de medicamentos fitoterápico e a notificação de produtos tradicionais fitoterápicos (Brasil, 2014).

O país apresenta políticas públicas direcionadas para o emprego de plantas medicinais em âmbito de saúde pública. A Política e Programa Nacional de Plantas Medicinais e Fitoterápicos (PNPMF) e a Política Nacional de Práticas Integrativas e Complementares (PNPIC) no SUS reforçam a ideia não apenas do uso de plantas medicinais, mas do seu uso racional, pois, diferente do pensamento que as plantas medicinais são inofensivas, sabe-se que elas são responsáveis por intoxicações e reações adversas (Brasil, 2006a; Brasil, 2016b). Entendendo a importância de se conhecer mais o potencial das plantas medicinais brasileiras, o professor Dr. Francisco de Matos da Universidade Federal do Ceará, criou o projeto intitulado farmácia viva que tem como objetivo principal incentivar o uso de plantas medicinais locais, bem como os medicamentos preparados a partir delas (Rufino et al., 2018). 
O Sistema Único de Saúde (SUS) apresenta uma lista com a relação nacional de plantas medicinais de interesse ao SUS. Esse é um ganho imenso principalmente a saúde que passa a ter acesso a mais opções terapêuticas com segurança (França et al., 2008 \& Figueredo et al., 2014). Dentro desse contexto, foi elaborada a seguinte questão que norteou esta pesquisa: Quais são os saberes e práticas dos usuários sobre as plantas medicinais no cuidado com a saúde e a importância da inserção da Fitoterapia no SUS? O objetivo desse trabalho foi de analisar a percepção de enfermeiros e usuários das Equipes de Saúde da Família Município de Juína-MT, sobre a inserção da fitoterapia como opção de tratamento e prevenção de doenças aos pacientes no SUS.

\section{METODOLOGIA}

A Utilizou-se o estudo de corte transversal, realizando entrevistas do tipo descritiva e estruturada para as coletas dos dados, utilizando perguntas abertas e fechadas categorizadas dentro das variáveis sociodemográficas e etnobotânica, com intuito de coletar informações e assim possibilitar a descrição do conhecimento, com amostragem aleatória simples, por sorteio, conforme descreve Godoy (2005). As questões norteadoras foram: Você considera a fitoterapia uma prática importante de uso; Com quem aprendeu sobre o uso da planta medicinal, Qual (is) planta (s) medicinal (is) você conhece? Como obtém a planta medicinal para uso? A pesquisa foi realizada nas quinze (15) Equipes de Saúde da Família do município de Juína-MT (Fig. 1). Foram entrevistados doze (12) enfermeiros e cento e trinta e oito (138) usuários selecionados por sorteio, usando o Microsoft Excel.

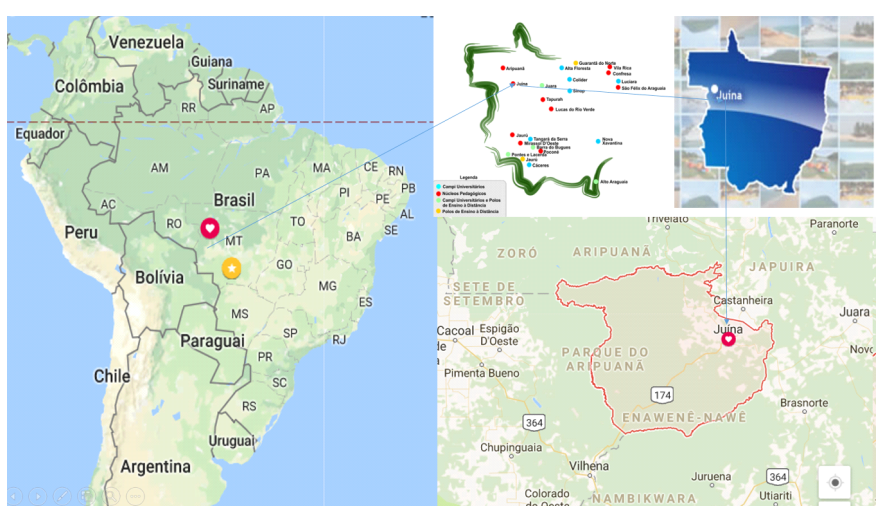

Figura 1. Localização do município de Juína, MT 
A pesquisa qualitativa possibilitou coletar informações e assim realizar análise do conhecimento sobre, as principais plantas e enfermidades por elas tratadas e respondendo às perguntas norteadoras da pesquisa, permitindo realizar a comparação imediata e análise dos resultados gerados (Scheaffer, Mendenhall, 1987 \& Bardin, 2004).

A pesquisa foi realizada no período de setembro a outubro/2017, sendo realizada em duas etapas, na primeira etapa foi feito o contato com todos os enfermeiros e os usuários atendidos nas respectivas Estratégia de Saúde da Família (ESF) e assim aos participantes foram informados sobre os objetivos da pesquisa e após todos os esclarecimentos foram agendadas as entrevistas. Após o agendamento, iniciou-se a segunda etapa, com a assinatura do Termo de Consentimento Livre Esclarecido (TCLE) e entrevistas na própria ESF utilizando um formulário semiestruturado e um gravador para melhor precisão da descrição das respostas as perguntas abertas sobre as plantas medicinais.

Os critérios de inclusão da pesquisa foram serem enfermeiros atuantes nas ESF do município de Juína e usuários cadastrados em suas respectivas ESF durante a visita do pesquisador. A idade dos participantes foi estabelecida como $\geq 18$ anos, deveriam ser moradores de Juína e aqueles que aceitaram assinar TCLE. Os critérios de exclusão foram todos aqueles que não se enquadravam nos critérios de inclusão e aqueles que que não apresentavam condições mentais para compreender e assinar o TCLE.

A pesquisa foi autorizada pelo Comitê de Ética em Pesquisa com Seres Humanos (CEP) da AJES, sob $n^{\circ}$ CAAE 69017317.6.0000.8099, seguindo as normas bioéticas e diretrizes aplicáveis a estudos envolvendo seres humanos (Resolução n ${ }^{\circ}$ 466/2012 do Conselho Nacional de Saúde Conselho), com a autorização da Secretária Municipal de Saúde de Juína e o Diretor Geral da Faculdade AJES. Durante a pesquisa a equipe garantiu todos os direitos éticos de pesquisa, onde todos os participantes tiveram total liberdade em desistir da pesquisa a qualquer momento.

\section{RESULTADO E DISCUSSÃO}

Com a execução dessa pesquisa foi possível entrevistas com 150 informantes, sendo 12 enfermeiros e com 138 usuários atendidos nas ESF. Observou-se que os doze enfermeiros que participaram da pesquisa, 11 era mulheres e 1 era homem, com idade entre 34 a 55 anos e dos 138 usuários 103 eram mulheres e 35 eram homens, a maioria natural do sul do Brasil, com idade entre 21 a 90 anos. 
Nas perguntas abertas utilizada para nortear percepção dos participantes destacam-se, (1) considerar a fitoterapia uma prática importante de uso, (2) com quem aprendeu sobre o uso da planta medicinal, (3) qual (is) planta (s) medicinal (is) você conhece e (4) como obtém a planta medicinal para uso.

Tanto os enfermeiros quanto os usuários informaram ser importante a inserção da fitoterapia no SUS, corroborando com a pesquisa realizada por Barreto (2011) \& Galavote et al. (2016) sobre a visão dos profissionais da ESF, onde resultados demonstram que os profissionais percebem a importância da inserção da fitoterapia no SUS, principalmente na doenças da atenção primária da saúde (APS).

A maioria dos enfermeiros informaram ter baixo conhecimento da fitoterapia (33\%), isso tem haver com a ausência da disciplina da graduação, pois todos os enfermeiros da pesquisa finalizaram sua graduação antes de 2009, ano que foi aprovado o Programa Nacional de Plantas Medicinais e Fitoterápicos no SUS e por isso, o baixo conhecimento sobre fitoterapia na graduação, corroborando com os achados de Terra et al. (2005), que também descreve a falta de informação dos enfermeiros e demais profissionais de saúde sobre a fitoterapia durante a graduação.

Porém, mesmo seus conhecimentos não sendo suficientes para a correta prescrição e/ou orientação de fitoterápicos aos usuários, os mesmos veem a fitoterapia como um tratamento complementar ou coadjuvante ao tratamento oficial com medicamentos sintéticos e apoiam a inserção da fitoterapia no SUS, com a capacitação aos profissionais de saúde nessa área, pois isso pode ser a principal dificuldade de inserção e avanços da fitoterapia no SUS do Brasil corroborando com Bastos \& Lopes (2010) que também entrevistaram enfermeiros das ESF para verificar o conhecimento sobre a fitoterapia verificando sua importância para muitos problemas a serem tratados (Nunes et al., 2006 \& Guimarães et al., 2006).

Portanto recomenda-se que seja incluída a disciplina de fitoterapia na graduação de enfermagem, isso vem ao encontro com o estudo de Feitosa et al. (2016), onde relata a opinião de acadêmicos sobre a inserção do conteúdo fitoterapia nos cursos de graduação na área da saúde sobre a inserção do conteúdo plantas medicinais e fitoterápicos (Faruque et al., 2019).

Na fala da maioria dos enfermeiros das Equipes de Saúde da Família de Juína a opinião é unânime quanto a importância da fitoterapia: 
"Acho que a fitoterapia deveria ser uma disciplina obrigatória na grade curricular de todos os profissionais da saúde no Brasil" (Enfermeiro 1).

"Sabemos da importância do saber milenar sobre as plantas medicinais, pois são práticas que existem na humanidade, a milhares de anos, e seu uso oficialmente, trará muitos benefícios a todos" (Enfermeiro 2).

"A população aqui da unidade sempre informa fazer uso de alguma planta para tratar alguma enfermidade, então acho importante sim a implantação da Fitoterapia, pois com certeza contribuirá muito para a melhoria dos serviços de saúde" (Enfermeiro 3).

“Eu acho importante a inserção da Fitoterapia no SUS" (Enfermeiro 4, 5, 9).

"Acredito muito no poder de cura das plantas medicinais e sua inserção contribuiria muito com os tratamentos das enfermidades da atenção básica" (Enfermeiro 14).

"Sei que a origem de muitos medicamentos sintéticos veio por meio das plantas medicinais, então a inserção da fitoterapia só contribuirá com a melhoria da saúde da população" (Enfermeiro 6).

Grande parte dos profissionais do Brasil considerarem importante a inserção da fitoterapia na atenção básica do SUS existe um enorme descaso por falta dos gestores do SUS (Antonio et al., 2013 \& Lopes, 2010).

$\mathrm{Na}$ fala dos usuários também encontramos afirmações de grande importância da implantação da fitoterapia no SUS de Juína.

"Uso plantas medicinais desde que nasci e sempre tive ótima saúde, pouco precisei usar medicamento de farmácia, então se um dia pudermos ser tratados na saúde pública com essa prática de cura será muito bom" (Usuário 1).

"Com certeza a implantação da Fitoterapia só ajudará, tanto a população quanto os profissionais" (Usuário 2).

"O uso correto das ervas só ajuda na cura e prevenção das doenças, então seria ótimo se o médico do SUS prescrevesse também" (Usuário 3).

Sobre a origem do conhecimento observa-se que a maioria informou conhecer as plantas medicinais com alguém da própria família, tanto os enfermeiros, quanto os usuários da saúde, isso corrobora com os achados de Kffuri (2008), Fontenel (2013) \& Mendieta et al. (2014), onde de 70 a $85 \%$ dos participantes afirmaram terem aprendido sobre plantas com as mães e as avós.

"Sempre via minha avó fazendo o chá e oferecendo a quem estava com alguma enfermidade, mais não achava que tinha tão importante ação, somente durante a faculdade foi perceber que um simples chá é um poderoso remédio pois ali tem muitos componentes químicos" (Enfermeiro 12). 
"Minha avó paterna era descendente de índio e sempre que tínhamos algum problema de saúde ela logo preparava ou pedia para nossa mãe preparar um chá, xarope ou tintura" (Usuário 29).

"Minha mãe sempre prepara o chá de alho para gripe de alguém na família, minha tia tem em casa o pé de crajiru uma planta muito usada para infecções urinárias, também usa a caninha-do-brejo para problemas renais" (Usuário 97).

O acesso a diferentes terapias medicamentosas está implícito nos princípios do SUS. A fitoterapia, por ser uma prática tradicional de saúde e já revelada em diversos estudos como de uso para fins terapêuticos para uma parcela significativa da população, poderia atender muitas das demandas de saúde da população usuária deste serviço (Tomazzoni, 2004 \& Almubayedh, Ahmad, 2020).

Apesar do Brasil possuir cerca de 350-550 mil espécies vegetais, aproximadamente 500 espécies constam na literatura como plantas medicinais utilizadas pela comunidade, isso pode ser um forte gargalo para que os gestores não valorizem esse importante conhecimento (Feitoza, 2006; Bieski; Guarim, 2018).

A população tem buscado cada vez mais o uso de práticas complementares para tratar doenças (Bruning et al., 2012; Thompson et al., 2019), objetivando obter menores efeitos colaterais e menor custo, com isso as plantas medicinais vem sendo amplamente usada pela população e estudada por pesquisadores para garantir sempre mais o uso seguro e eficaz (Barreto, 2011 \& Sampaio et al., 2013).

As informações obtidas apontaram que 94\% dos enfermeiros e $94 \%$ dos usuários entrevistados fazem uso de alguma planta medicinal e quando precisam utilizar alguma planta pegam no próprio quintal (68\%). A maior finalidade da utilização da planta medicinal é para fins curativos $(66 \%)$.

O total das espécies citadas da pesquisa perfizeram 70 espécies, pertencentes a 41 famílias botânicas conforme com destaque as famílias Lameaceae (11\%). Esse número é considerado muito baixo comparado com outro estudo realizado em Juína (Bieski, 2015). Provavelmente isso tenha ocorrido pelo fato das entrevistas terem sidos realizadas nas unidades de saúde e não em suas residências, enquanto que na pesquisa conduzida por Bieski (2015), as entrevistas foram pré-agendadas nas residências dos usuários, mostrando a grande interferência das informações. Apesar dos usuários conhecerem mais plantas que os enfermeiros o local da entrevista pode ter prejudicado o número de plantas citadas. 
Observa-se a percepção da maioria dos informantes em sua maioria demonstram que as plantas medicinais tem importante finalidade curativa $(66 \%)$, cintando tratar infeções urinárias, pedra nos rins, problemas estomacais, diabetes, hipertensão, cicatrização, ansiedade, infecções vaginais, inflamação na garganta, gripe e no útero com as plantas medicinais, são elas Allium sativum L (alho), Arrabidaea chica (Bonpl.) B. Verl (crajiru), Costus spicatus (Jacq.) Sw (cana-do-brejo), Curcuma longa L. (açafrão), Gossypium hirsutum L. (algodão), Paullinia cupana Kunth (guarana), Plectranthus barbatus Andrews (boldo-da-terra), Psidium guajava L. (goiabeira), Syzygium cumini Lam. (jambolão).

As variedades de plantas medicinais utilizadas pela população em diversos usos se devem ao elevando número de metabólitos ativos presentes nas mesmas (Almubayedh \& Ahmad, 2020).

Com base nas plantas medicinais mais citadas é importante verificar as falas dos informantes.

"O alho não pode faltar na casa de nenhuma pessoa, pois é uma planta que tem uma infinidade de beneficios, uso para gripe, pressão alta e baixar colesterol" (Usuário 89).

"Quando tenho infecção urinária logo faço chá de crajiru" (Usuário 140).

"Gosto de usar diferentes plantas medicinais para infecção urinária, uso crajiru, caninha-do-brejo, também uso as folhas de algodão para infecção no útero" (Usuário 123).

"Sempre indico o chá das folhas do Jambolão para quem tem diabetes, pois abaixa mesmo o diabetes e as pessoas param de tomar o medicamento pois tem muitos efeitos colaterais" (Usuário 139).

"Sempre me deparo com algum usuário falando que usou alguma planta medicinal e ficou bom, acho que deveríamos ter uma horta na unidade de saúde para fazermos oficinas e assim aprendermos mais sobre as mesmas" (Enfermeiro 8).

"Sei que existem muitos estudos que comprovam a eficácia do alho para várias doenças e por isso faço o uso na alimentação e quase não fico gripada" (Enfermeiro 7).

A maioria da população brasileira possui um saber significativo a respeito da utilização de plantas como, para tratar ou prevenir as enfermidades. As pesquisas realizadas com comunidades são muito importantes, pois carregam uma bagagem sobre as plantas medicinais, porém, esse saber está sofrendo ameaça constante devido à influência direta da medicina moderna e o avanço da tecnologia e, principalmente, pelo desinteresse das pessoas mais jovens, o que pode interromper o processo de transmissão do saber entre as gerações (Amorozo, 1996 \& Ferreira, 2018). 
As pesquisas etnobotânicas e etnofarmacológicas são importantes ferramentas de registro e documentação dos usos de plantas, principalmente da diversidade de enfermidades tratadas, gerando conhecimento útil ao desenvolvimento de novos medicamentos, à conservação da biodiversidade, a valorização do saber e da cultura local (Araújo, 2009). Os resultados indicam que os enfermeiros e os usuários da pesquisa têm bom conhecimento sobre as plantas medicinais, sendo a maioria espécies exóticas e de fácil cultivo nos quintais, isso pode estar ocorrendo devido a maioria dos informantes serem da área urbana, inclusive muitas das plantas medicinais, já são regulamentadas pelo ANVISA, corroborando com os estudos de Bieski (2010) \& Fontenele et al. (2013) que relatam em suas pesquisas a predominância das espécies exóticas, serem cultivadas nos quintais, enquanto as espécies nativas serem colhidas no mato.

\section{CONCLUSÕES}

Com essa pesquisa foi possível verificar que a maioria dos usuários e enfermeiros, conhecem ou usam alguma planta medicinal e trazem esse conhecimento de suas raízes familiares, pois as perguntas abertas que nortearam a percepção dos participantes destacam-se, considerar a fitoterapia uma prática importante de uso, com quem aprendeu sobre o uso da planta medicinal, qual (is) planta (s) medicinal (is) você conhece e como obtém a planta medicinal para uso. Na fala da maioria dos enfermeiros das Equipes de Saúde da Família de Juína a opinião é unânime quanto a importância da fitoterapia, onde as plantas medicinais podem ser utilizadas com segurança e eficácia, conforme recomendações do Ministério da Saúde e documentos regulatórios, contribuindo assim com a inserção e efetivação das políticas públicas do SUS, além de atividades de educação continuada com a comunidade e profissionais, suprindo a carência de formação técnicocientífica da equipe com relação aos fitoterápicos, um dos empecilhos para a implantação da fitoterapia no SUS.

Embora os registros etnobotânicos sirvam como repositório de conhecimento, eles não são necessariamente uma representação completa do uso atual de plantas medicinais, com ele foi possível verificar as enfermidades mais tratadas com plantas medicinais no município de Juína.

Observa-se que algumas plantas sejam usadas predominantemente que outras, onde observou-se que as plantas medicinais para tratar infecções urinárias, pedra nos rins, problemas estomacais, diabetes, hipertensão, cicatrização, ansiedade, infecções vaginais, 
inflamação na garganta e no útero, especificamente aquelas com maior abundância e facilidade de obtenção conhecidas por serem seguras e eficazes. Ao pesquisar a comunidade por meio de entrevistas observou-se que as plantas medicinais mais usadas foram, Allium sativum L. (alho), Fredericia chica (Bonpl.) B. Verl (Crajiru), Costus spicatus (Jacq.) Sw. (caninha-do-brejo), Curcuma longa L. (açafrão), Gossypium hirsutum L. (algodão), Paullinia cupana Kunth. (guaraná), Plectranthus barbatus Andrews (boldo), Psidium guajava L. (goiaba), Syzygium cumini Lam. (jamelão). Todas elas ricas em compostos terpenos, compostos fenólicos e alcalóides plantas, algumas dessas plantas medicinais apresentam validação científica e estão disponíveis pela indústria farmacêutica.

Os enfermeiros (69\%) e usuários (96\%) avaliaram como muito interessante, a implantação do programa de plantas medicinais no SUS de Juína-MT, Brasil. A utilização de plantas medicinais vem ao encontro das proposições da Organização Mundial da Saúde (OMS), que incentiva a valorização das terapias tradicionais, reconhecidas como recursos terapêuticos úteis, podendo atender a algumas demandas de saúde da população, além de contribuir para o sistema local de saúde dos usuários do sistema público de saúde e com imensuráveis benefícios tanto para a população quanto para os gestores, principalmente na economia dos gastos com a doença. O conhecimento etnobotânico muito contribui com a efetivação das políticas nacionais e estadual de plantas medicinais e fitoterápicos no SUS e assim poderá assegurar a efetivação da promoção, proteção e recuperação da saúde da população de forma segura e eficaz.

Agradecimentos. Agradecemos a Faculdade Noroeste de Mato Grosso e a Secretaria Municipal de Saúde de Juína.

\section{REFERÊNCIAS}

Amorozo, M.C.M. (1996). A abordagem etnobotânica na pesquisa de plantas medicinais. In: Di Stasi, L.C. Plantas medicinais: arte e ciência. São Paulo: UNESP. p. 47-68.

Antonio, G. D., Tesser, C. D. \& Moretti-Pires, R. O. (2013). Contribuições das plantas medicinais para o cuidado e a promoção da saúde na atenção primária. Interface-Comunicação, Saúde, Educação, v. 17, n. 46, p. 615-633.

Almubayedh H. \& Ahmad R. (2020). Ethnopharmacology, phytochemistry, biological activities, and therapeutic applications of Cedrela serrata Royle: A mini review. J Ethnopharmacol. Jan 10; 246:112206.

Araújo, M. M. (2009). Estudo etnobotânico das plantas utilizadas como medicinais no Assentamento Santo Antonio, Cajazeiras, PB, Brasil. Cajazeiras, PB: UFCG. Dissertação de Mestrado, Universidade Federal de Campina Grande.

Bardin, L. (2004). Análise de conteúdo. Lisboa: Edições 70. 
Barreto, B. B. (2011). Fitoterapia na Atenção Primária à Saúde: a visão dos profissionais envolvido. 93 f.

Bastos, R. A. A., \& Lopes, A. M. C. (2010). A fitoterapia na Rede Básica de Saúde: o olhar da enfermagem. Rev. bras. ciênc. saúde, v. 14, n. 2

Brasil. (2010). Ministério da saúde. Institui a Farmácia Viva no âmbito do Sistema Único de Saúde (SUS). Brasília,

Brasil. (2006a). Ministério da Saúde. Portaria n 971, de 03 de maio de 2006. Aprova a Política Nacional de Práticas Integrativas e Complementares no SUS. Diário Oficial [da] República Federativa do Brasil, Poder Executivo, Brasília, DF, 4 mai. Seção 1, p 20.

Brasil. (2006b). Ministério da Saúde. Secretaria de Ciência, Tecnologia e Insumos Estratégicos. Departamento de Assistência Farmacêutica. Política e Programa Nacional de Plantas Medicinais e Fitoterápicos - Brasília: Ministério da Saúde. 190 p.

Brasil. (2014). Ministério da Saúde. Secretaria de Ciência, Tecnologia e Insumos Estratégicos. Departamento de Assistência Farmacêutica e Insumos Estratégicos. Serviços farmacêuticos na atenção básica à saúde Brasília: Ministério da Saúde, 108 p.: il. - (Cuidado farmacêutico na atenção básica; caderno 1).

Bieski, I. G. C., Guarim G. N. (2018). Conhecimentos Etnobotânico de Plantas Medicinais na Amazônia MatoGrossense - ISBN-10 859383602X, ISBN-1. R.b.e editora. p.157.

Bieski, I. G. C. (2010). Conhecimento etnofarmacobotânico de plantas medicinais utilizadas por comunidades tradicionais do Distrito Nossa Senhora Aparecida Chumbo, Poconé, Mato Grosso, Brasil. 298 p.

Bruning, M. C. R., Mosegui, G. B. G. \& Vianna, C. M. M. (2012). A utilização da fitoterapia e de plantas medicinais em unidades básicas de saúde nos municípios de Cascavel e Foz do Iguaçu-Paraná: a visão dos profissionais de saúde. Ciência \& Saúde Coletiva, v. 17, n. 10.

Demo, P. (2013). Metodologia do conhecimento científico. São Paulo: Atlas, 128p.

Feitosa, M. H. A., Soares, L. L., Borges, G. A., Magalhães. M., \& Andrade S. M. C. (2016). Inserção do Conteúdo Fitoterapia em Cursos da Área de Saúde Inclusion of Phytotherapy Content in Health Training Courses Revista Brasileira de Educação Médica 19740 (2): 197-203.

Ferreira, A. L. S. (2018). Etnofarmacobotânica e os saberes tradicionais na comunidade barreirinho, Santo Antônio do Leverger - MT, Brasil. Dissertação apresentada à Faculdade de Engenharia Florestal da Universidade Federal de Mato Grosso, como parte das exigências do Curso de Pós-Graduação em Ciências Florestais e Ambientais, para obtenção do título de mestre. Cuiabá, Mato Grosso, 109 pp.

França, I. S. I. S. X., Souza, J. A., Baptista, R. S., \& Britto, V. R. S. (2008). Medicina popular: benefícios e malefícios das plantas medicinais. Rev Bras Enferm, Brasília 2008 Rev Bras Enferm, Brasília, mar-abr; 61(2): 201-8

Figueredo, C. A. de.; Gurgel, I. G. D.; Junior-Gurgel, G. D. (2014) A Política Nacional de Plantas Medicinais e Fitoterápicos: construção, perspectivas e desafios 24(2): 381-340.

Fontenele, R. P., Sousa, D. M. P., Carvalho, A. L. M., \& Oliveira, F. A. (2013). Fitoterapia na Atenção Básica: olhares dos gestores e profissionais da Estratégia Saúde da Família de Teresina (PI), Brasil. Ciênc. saúde coletiva vol.18 n.8 Rio de Janeiro.

Faruque M.O., Feng G., Khan M.N.A., Barlow J.W., Ankhi U.R., Hu S., Kamaruzzaman M, Uddin S.B., Hu X. (2019) Qualitative and quantitative ethnobotanical study of the Pangkhua community in Bilaichari Upazilla, Rangamati District, Bangladesh. J Ethnobiol Ethnomed. Feb 5;15(1):8. doi: 10.1186/s13002-019-0287-2.

Khan A.A., Ali F., Ihsan M. (2015). Ethnobotanical study of the medicinal plants of Tehsil Charbagh, district Swat, Khyber Pakhtunkhwa, Pakistan. Am. Eurasian J. Agric. Environ. Sci. 15:1464-1474. 
Kffuri, C. W. (2008). Etnobotânica de plantas medicinais no município de Senador Firmino (Minas Gerais) [dissertação na internet]. Viçosa (MG): Universidade Federal de Viçosa. 140 pp.

Galavote. H. S; Zandonade. E; Garcia. A. C. P; Freitas. P. S. S; Seidl. H; Contarato. P. C; Andrade. M. A. C; \& Duarte. R.C. L. (2016). O trabalho do enfermeiro na atenção primária à Saúde. Esc Anna Nery; 20(1):90-98

Godoy, A. S. (2005). Refletindo sobre critérios de qualidade da pesquisa qualitativa. Revista Eletrônica de Gestão Organizacional, v. 3, n. 2, p. 81-89, mai. /ago.

Guimarães, J; Medeiros, J. C; \& Vieira, L. A. (2006). Programa Fitoterápico Farmácia Viva no SUS - Betim. ABFIT. pp 112.

Mendieta, M. C., Souza, A. D. Z., Vargas, N. R. C., Piriz, M. A., Echevarría-Guanilo, M. E., Heck, R. M. (2014). Transmissão de Conhecimento sobre Plantas Medicinais no Contexto Familiar: Revisão Integrativa. Rev Enferm UFPE on line., Recife, 8(10):3516-24, out.

Machado, O. (1945). Contribuiçao ao estudo das plantas medicinais do BrasiL. Maytenus obtusifolia Mart. Rodriguésia, v. 9, n. 18, p. 9-15.

Matos, F. J. A. (1985). Recuperação de informações, seleção e divulgação de plantas medicinais. Revista Brasileira de Farmácia, v. 66, n. 4, p. 49-61.

Nunes, J. D.; Maciel, M. V., Lima, J. P., Oliveira, M.J.R; Simões, M.J.S; \& Sassi, C.R.R. (2006). Fitoterapia no sistema de saúde pública (SUS) no Estado de São Paulo, Brasil. Revista Brasileira de Plantas Medicinais, Botucatu v. 8, n. 2, p. 39-41.

Rezende, H. A., \& Cocco, M. I. M. (2002). A utilização de fitoterapia no cotidiano de uma população rural. Rev. Esc. Enferm. USP [online], vol.36, n.3, pp.282-288.

Rufino, L. L.; Rojas-Gamarra, G.; Bandeira, M.A.B.; Souza, J. R.F. de.; Reis, J. N.P. (2018). Prática das farmácias vivas do município de Fortaleza, Ceará, e a necessidade de uma ação de extensão sistemática. Extensão Rural, vol. 25, n.4, pp. 40-53.

Santana, J. D., Suzy Hellen A. Dourado, S. H. A. Bieski, I. G. C. (2018). Potencial das Plantas Medicinais no Tratamento de Doença de Alzheimer com Enfase em Curcuma longa. Revista Saúde Viva Multidisciplinar da AJES, v. 1, n. 1, p. 1 - 156, Ago./Dez.

Sampaio, L. A., Oliveira, A. R., Kerntopf, M. R., Júnior, F., E. B., \& Menezes, I. R. A. (2013). Percepção dos enfermeiros da estratégia saúde da família sobre o uso da fitoterapia. Revista Mineira de Enfermagem, v. 17 , n. 1 , p. $77-85$.

Scheaffer, R.L., Mendenhall, W. \& Ott, L. Elementos de Muestreo. Grupo editorial Iberoamericana S. A. México, 1987, 321p.

Terra, I. A. (2005). Ensino de Botânica nos cursos de graduação em farmácia: sua contribuição na formação e atuação do farmacêutico. Teses e Dissertações PPGECIM. P. 117.

Tomazzoni, M. I., Negrelle, R. R. B. \& Centa, M. L. (2004). Fitoterapia popular: a busca instrumental enquanto prática terapêutica Revista Texto \& Contexto Enfermagem, vol. 15, núm. 1, janeiro-março, pp. 115-121.

Thompson, A., Munkara, G., Kantilla, M., Tipungwuti, J. (2019) Medicinal plant use in two Tiwi Island communities: a qualitative research study. J Ethnobiol Ethnomed. 15: 40. 